\title{
Facial nerve palsy in otitis externa: A red flag?
}

\author{
Aliyya Badaruddin, May May Choo \\ Badaruddin A, Choo MM. Facial nerve palsy in otitis externa: A red Flag?. Malays Fam Physician. 2021;16(1).
}

\section{Keywords:}

necrotizing otitis externa,

malignant otitis externa,

facial nerve palsy

\section{Authors:}

\section{Aliyya Badaruddin \\ (Corresponding author) \\ MB, BCh, BAO, MOphthal \\ Department of Ophthalmology \\ University of Malaya Medical Centre \\ Kuala Lumpur, Malaysia \\ Email: aliyyabadaruddin@gmail.com}

\section{Choo May May}

FRCS

Department of Ophthalmology University of Malaya Medical Centre Kuala Lumpur, Malaysia

\section{Abstract}

Otitis externa is an infection of the external auditory canal. It rarely results in facial palsy except in severe cases such as necrotizing otitis externa, which is a life-threatening invasive infection of the external auditory canal. Early recognition with prompt and appropriate treatment of necrotizing otitis externa is crucial to prevent more sinister complications. Here we report a case of an elderly gentleman who presented with otitis externa and developed facial palsy a month later. We identified possible problems that may have led to the complication so that such an occurrence can be prevented in the future.

\section{Introduction}

Facial palsy is an uncommon complication of otitis externa. It is more commonly seen in necrotizing otitis externa (NOE) with skull base osteomyelitis, especially if comorbidities are present. ${ }^{1}$ Here we report a case of an elderly diabetic patient who presented with otitis externa and subsequently developed facial palsy. We identified the possible problems that may have led to this complication.

\section{Case Report}

A 65-year-old man presented with right otalgia and otorrhoea for a duration of 2 days. He described the pain as excruciating and as sufficiently bad to disturb his sleep and disrupt some of his daily activities. He was diagnosed

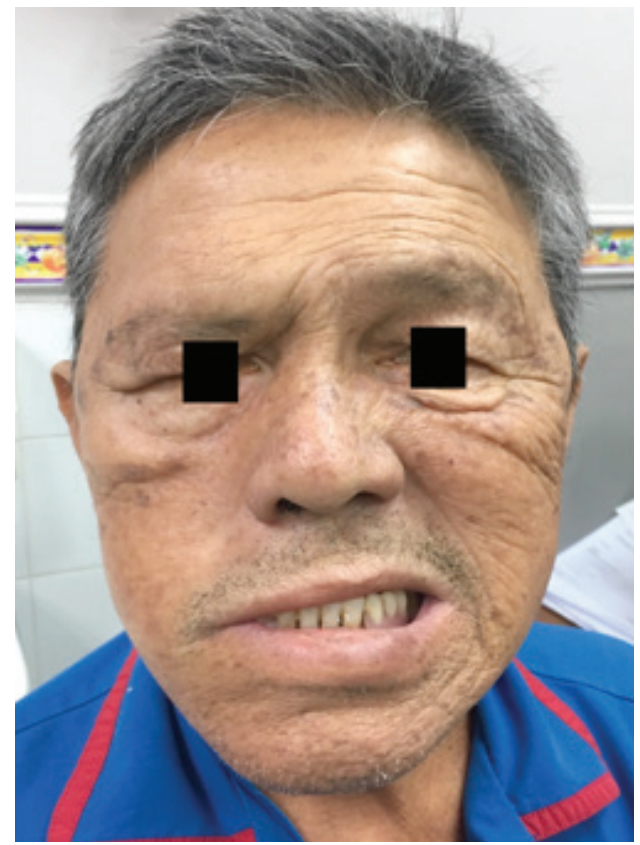

Figure 1: Right lower motor neurone facial palsy with right acute otitis externa by a general practitioner and completed 2 courses of oral antibiotics in addition to topical antibiotic. However, the pain did not resolve.

Four weeks later he developed facial asymmetry with drooling of saliva and was urgently referred to a neurologist to rule out a central cause. On examination, he had right lower motor neuron facial palsy with loss of right nasolabial fold, drooping of the corner of the mouth, right lagophthalmos and loss of right forehead wrinkles (Figure 1).

He was then referred to the otorhinolaryngology team who discovered right severe otitis externa with granulation tissue in the external auditory canal. Right ear toileting and swab were performed, which

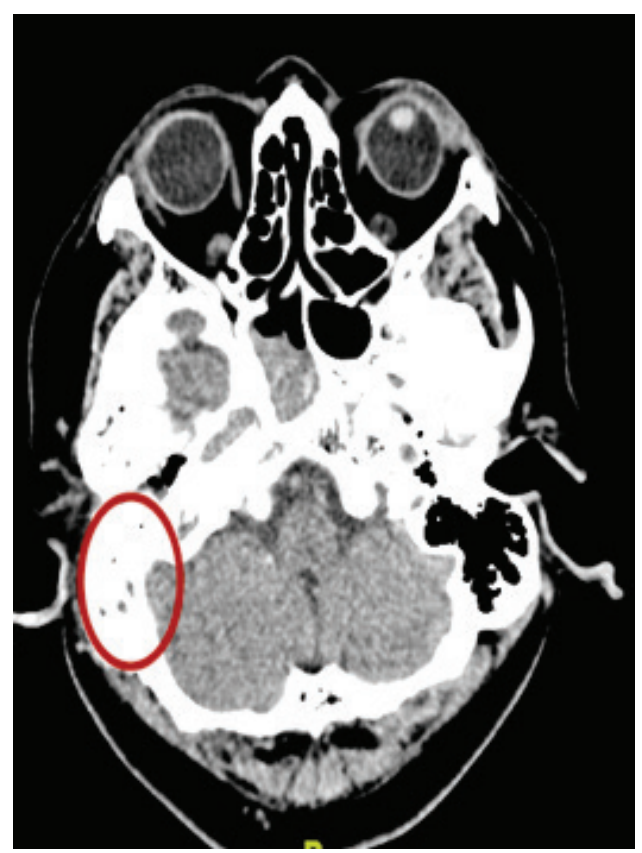

Figure 2: CT brain showing right mastoiditis and base of skull erosion 
revealed Candida albicans. He was started on clotrimazole ear spray. He underwent High Resolution Computed Tomography of the temporal bone, which showed right mastoiditis and base of skull erosion (Figure 2). A biopsy ruled out malignant tumors. A subsequent Magnetic Resonance Imaging (MRI) showed enhancement at the right mastoid with no intracranial metastases (Figure 3). A final diagnosis of right necrotizing otitis externa with base of skull osteomyelitis and secondary facial palsy was made. He was started on intravenous ceftazidime $1 \mathrm{~g}$ every 8 hours, intravenous dexamethasone $8 \mathrm{mg}$ (in tapering doses) and underwent mastoidectomy (Figure 4).

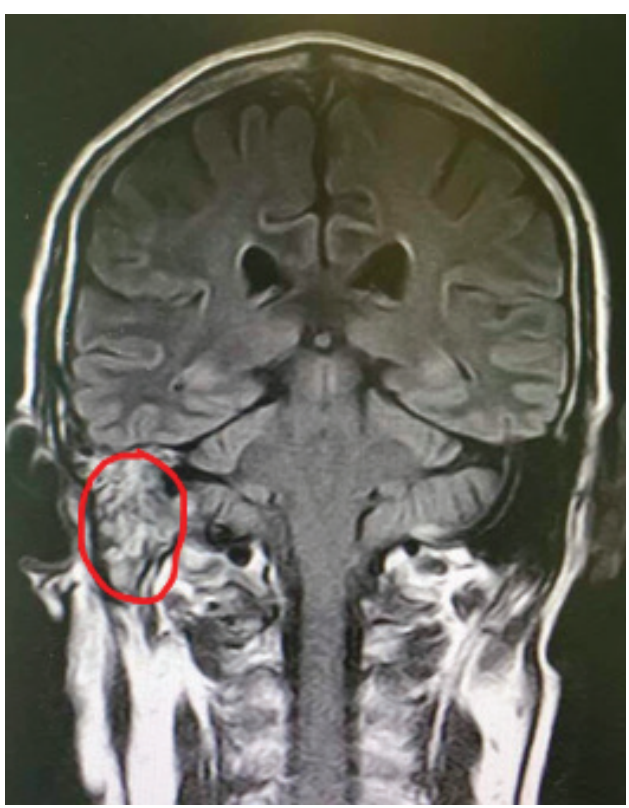

Figure 3: MRI brain/neck showing right mastoiditis

\section{Discussion}

Facial palsy is a rare complication of otitis externa. Previous studies have reported on facial palsy occurring in necrotizing otitis externa, especially in immunocompromised individuals such as diabetic patients. ${ }^{2,3}$ Diabetics have an increased $\mathrm{pH}$ in cerumen which predisposes them to infection such as Pseudomonas aeruginosa. The endarteritis and microangiopathy in diabetics leads to hypoperfusion in the subcutaneous tissue. ${ }^{1,4,5}$

Necrotizing otitis externa (NOE) associated with skull base osteomyelitis is an uncommon, rapidly spreading, life-threatening invasive infection of the external auditory canal (EAC) and the lateral skull base. ${ }^{1}$ Granulation tissue in the external auditory canal is a
Ophthalmic examination revealed right lagophthalmos with good Bell's reflex. There was mild punctate epithelial erosion. He was prescribed with lubricants and taping the right eye when asleep. The patient has had underlying diabetes for the past 8 years, which was not well-controlled with oral metformin $1 \mathrm{~g}$ and gliclazide $80 \mathrm{mg}$ twice daily. His random blood sugar was $13.0 \mathrm{mmol} / \mathrm{L}$ and $\mathrm{HbA} 1 \mathrm{C}$ $8 \%$. He was referred for optimization of sugar control while in the ward. Subcutaneous Insulatard $10 \mathrm{U}$ was added after which his blood sugar has been within normal range. There was gradual improvement of his facial palsy following rehabilitation including physiotherapy.

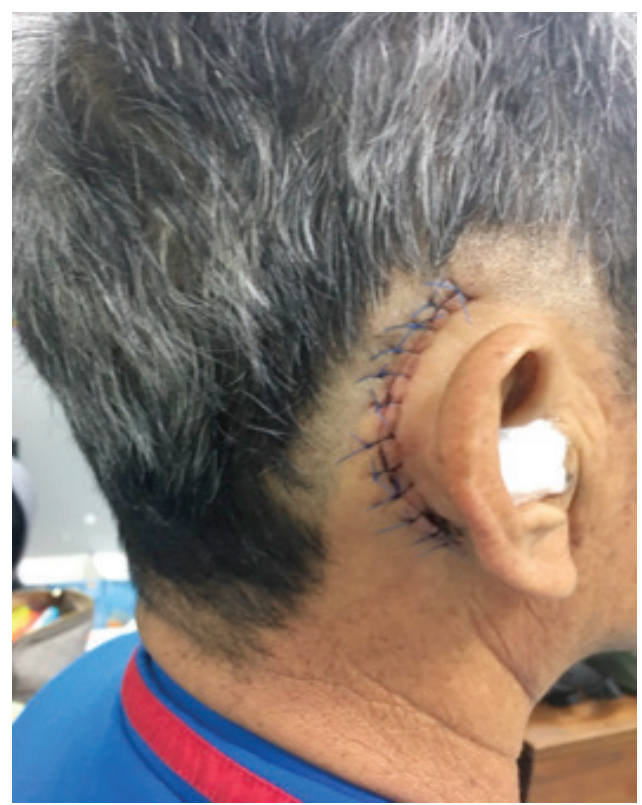

Figure 4: Right cortical mastoidectomy scar

pathognomonic finding of NOE. ${ }^{6}$ The disease may spread through the fissures of Santorini to the adjoining skull base, causing periostitis, osteomyelitis and cranial nerve palsies. ${ }^{1}$ It is very important to identify the disease and treat it early as it can cause more sinister complications such as meningitis, brain abscess and death. Treatment of NOE includes immunosuppression, local treatment of auditory canal and long-term antibiotic therapy (approximately 6-8 weeks). ${ }^{1,3}$ The role of surgery in NOE is limited; mastoidectomy is performed if mastoid air cells are involved and in persistent otalgia, a transection of $\mathrm{CN} \mathrm{V}$ and CN IX has been reported. ${ }^{3,7}$ Strict glycemic control is mandatory. ${ }^{3}$

Acute otitis externa (AOE) and NOE may have similar presentation in the beginning. However, 
in NOE the pain is so excruciating that it may disturb one's sleep and in later stages it can cause complications such as osteomyelitis of the skull with cranial nerves involvement or death from disseminated septic thrombo-emboli of the brain. Examination of NOE may show granulation tissue in EAC at the bone-cartilage junction. Imaging studies to confirm $\mathrm{NOE}$ include Computed Tomographic (CT) and bone scanning. Unlike AOE, NOE requires long-term intravenous antibiotic therapy. 3,8 EAC cholesteatoma is an inflammatory lesion of the temporal bone with CT findings of soft tissue mass in EAC and adjacent bone erosion.'

There are several mechanisms thought to cause facial palsy in NOE. The primary mechanism is direct involvement of the nerve by toxins from the pathogens. Infection may spread through Santorini fissures to the base of the skull, causing bone destruction before spreading medially, thus triggering facial palsy. ${ }^{2,3}$ When facial palsy appears late in the disease, it may be due to silent mastoiditis, which may only be diagnosed with brain imaging. The presence of facial palsy indicates a poorer prognosis due to the deep penetration along the base of the skull. This may put other cranial nerve palsies at high risk and, due to the poor perfusion of the diseased area, a longer intravenous antibiotic treatment is required. ${ }^{3,10}$

Here we present a case of right facial palsy in a patient who was initially diagnosed with acute otitis externa which did not show improvement despite being treated with topical and oral antibiotics. We identified the possible causes that may have led to the complication of facial palsy. Firstly, when the patient initially presented with otalgia and otorrhoea, it is important to identify the causes and to start appropriate treatments. Ear secretions should be cultured prior to starting medications as early treatment with appropriate antimicrobials may achieve good cure rates and reduce the associated morbidity/mortality. ${ }^{1}$ Furthermore, the pattern of cultures may change after treatment has been initiated. ${ }^{3}$ If the infection is not responsive to antibiotics, a fungal infection should be considered. More importantly, early suspicion of NOE should be made if the symptoms remain persistent despite multiple courses of antibiotics. Hence, it is important to perform a good otoscopic examination and look for the presence of granulation tissue, especially in severe otalgia cases, for which a prompt referral to the otorhinolaryngology team should be made. ${ }^{1,10}$ Treating NOE with oral antibiotic alone as an outpatient is often inadequate; instead a long-term intravenous antibiotic is required. ${ }^{1,3}$

When patients with otitis externa went on to develop facial palsy, subsequent management of the problem may have been delayed by unnecessary referrals. This patient with lower motor neuron facial palsy associated with otalgia and otorrhoea was referred to the neurologist prior to seeing the otorhinolaryngology team, as it is crucial to exclude NOE. This, however, may have caused a delay in the treatment if the patient was not referred to the appropriate team promptly and may have resulted in more serious complications, including death. Therefore, a thorough examination must be performed to look for the causes of facial palsy in the ears, mastoid region, oral cavity, eyes, scalp, parotid glands and a complete neurological examination must be performed too before making a referral.

All patients with comorbidities including diabetes should be managed with a holistic approach. Treatment should not just focus on the otitis externa but also on any underlying health issues. The patient's sugar control should have been managed concurrently with the otitis when his case was first presented, as diabetic patients are more susceptible to a wide range of diseases including NOE. Medical experts should be involved earlier to co-manage any underlying comorbidities. In this case, if NOE had been diagnosed earlier during his initial presentation at the primary care level, the complication of facial nerve palsy may have possibly been avoided. A prompt referral and treatment in NOE cases may avoid other more serious complications including death.

\section{Conclusion}

Facial nerve palsy in otitis externa is an uncommon complication that may be prevented with proper management. This includes early recognition of the disease, early referrals, appropriate treatments and optimizing the comorbidities. Necrotizing otitis externa must be suspected in otitis externa cases in elderly diabetic patients who do not respond to oral antibiotic treatments.

\section{Funds Declaration}

No funds were received for this case report. 


\section{Patient Consent}

Written informed consent was obtained from the patient for this case report and its accompanying images.

\section{Disclosure of interest}

The authors report no conflicts of interest.

\section{How does this paper make a difference to general practice?}

- It creates awareness among clinicians and health staff of the need to recognize and differentiate necrotizing otitis externa (NOE) from acute otitis externa.

- It describes a case commonly encountered in a general-practice setting which may have been overlooked as a mild case but was ultimately discovered to be more complicated.

- A high index of suspicion for NOE is important when dealing with otitis externa not responding to topical/oral antibiotics.

\section{References}

1. Kumar SP, Ravikumar A, Somu L, Ismail NM. Malignant otitis externa: an emerging scourge. Journal of Clinical Gerontology and Geriatrics. 2013;4:128-131.

2. Walton J, Coulson C. Fungal malignant otitis externa with facial nerve palsy: tissue biopsy aids diagnosis. Case Rep Otolaryngol. 2014;2014:192318

3. Handzel O, Halperin D. Necrotizing (malignant) external otitis. Am Fam Physician. 2003; 68:309-312.

4. Singh A, Khabori M, Hyder J. Skull base osteomyelitis: diagnostic and therapeutic challenges in atypical presentation. Otolaryngol Head Neck Surg. 2005;133:121-125.
5. Illing E, Zolotar M, Ross E, Olaleye O, Molony N. Malignant otitis externa with skull base osteomyelitis. J Surg Case Rep. 2011;2011(5):6

6. Roberts JR. Malignant externa otitis: disastrous for diabetics. Emergency Medicine News. 2004;26:24-26.

7. Chandler JR. Pathogenesis and treatment of facial paralysis due to malignant external otitis. Ann Otol Rhinol Laryngol. 1972;81:648-658.

8. Cohen D, Friedman P, Filon A. Malignant external otitis versus acute external otitis. J Laryngol Otol. 1987;101: 211-215.
9. Heilbrun ME, Salzman KL, Glastonbury CM, et al. External auditory canal cholesteatoma: clinical and imaging spectrum. Am J Neuroradiol. 2003; 24: 751-756.

10. Cohen D, Friedman P. The diagnostic criteria of malignant external otitis. J Laryngol Otol. 1987;101:216-21. 